\title{
Cellular uptake and biocompatibility of bismuth ferrite harmonic advanced nanoparticles
}

Davide Staedler ${ }^{\mathrm{a}, 1}$, Solène Passemard, MSc, PhD ${ }^{\mathrm{a}, 1}$, Thibaud Magouroux, MSc, $\mathrm{PhD}^{\mathrm{b}}$, Andrii Rogov, $\mathrm{MSc}^{\mathrm{b}}$, Ciaran Manus Maguire, $\mathrm{MSc}^{\mathrm{c}}$, Bashir M. Mohamed, $\mathrm{PhD}^{\mathrm{c}}$, Sebastian Schwung, MSc ${ }^{\mathrm{d}}$, Daniel Rytz, MSc, $\mathrm{PhD}^{\mathrm{d}}$, Thomas Jüstel, MS, $\mathrm{PhD}^{\mathrm{e}}$, Stéphanie Hwu, MSc ${ }^{b}$, Yannick Mugnier, MSc, PhD ${ }^{\mathrm{f}}$, Ronan Le Dantec, MSc, PhD ${ }^{\mathrm{f}}$, Yuri Volkov, MD, $\mathrm{PhD}^{\mathrm{c}, \mathrm{g}}$, Sandrine Gerber-Lemaire, $\mathrm{MSc}, \mathrm{PhD}^{\mathrm{a}}$, Adriele Prina-Mello, MSc, $\mathrm{PhD}^{\mathrm{c}, \mathrm{g}}$, Luigi Bonacina, MSc, $\mathrm{PhD}^{\mathrm{b}, *}$, Jean-Pierre Wolf, MSc, $\mathrm{PhD}^{\mathrm{b}}$

${ }^{a}$ Institute of Chemical Sciences and Engineering, EPFL, Batochime, 1015, Lausanne, Switzerland

${ }^{\mathrm{b}}$ GAP-Biophotonics, Université de Genève, 22 Chemin de Pinchat, 1211 Genève 4, Switzerland

${ }^{\mathrm{c}}$ Nanomedicine Laboratory and Molecular Imaging Group, School of Medicine, Trinity Centre for Health Sciences, Trinity College, D8, Dublin, Ireland

${ }^{\mathrm{d}}$ FEE Gmbh, Struthstrasse 2, 55743 Idar-Oberstein, Germany

${ }^{\mathrm{e}}$ Fachbereich Chemieingenieurwesen, Fachhochschule Münster, Stegerwaldstrasse 39, 48565 Steinfurt, Germany

${ }^{\mathrm{f}}$ Univ. Savoie, SYMME, F-74000, Annecy, France

${ }^{\mathrm{g}}$ AMBER Centre and CRANN Institute, Trinity College, D2, Dublin, Ireland

Received 17 August 2014; accepted 22 December 2014

\begin{abstract}
Bismuth Ferrite (BFO) nanoparticles (BFO-NP) display interesting optical (nonlinear response) and magnetic properties which make them amenable for bio-oriented diagnostic applications as intra- and extra membrane contrast agents. Due to the relatively recent availability of this material in well dispersed nanometric form, its biocompatibility was not known to date. In this study, we present a thorough assessment of the effects of in vitro exposure of human adenocarcinoma (A549), lung squamous carcinoma (NCI-H520), and acute monocytic leukemia (THP-1) cell lines to uncoated and poly(ethylene glycol)-coated BFO-NP in the form of cytotoxicity, haemolytic response and biocompatibility. Our results support the attractiveness of the functional-BFO towards biomedical applications focused on advanced diagnostic imaging.
\end{abstract}

(C) 2015 Elsevier Inc. All rights reserved.

Key words: Nanophotonic; Non-linear imaging; Bismuth ferrite; PEGylation; Biocompatibility

Funded by: Partially funded by European Commission funded project NAMDIATREAM project (FP7 LSP ref 246479) and CAN project (European Regional Development Fund through the Ireland Wales Programme 2007-13 INTERREG 4A). The study was performed in the context of the European COST Action MP1302 Nanospectroscopy.

Conflicts of interest: the authors declare no conflict of interest.

*Corresponding author.

E-mail address: luigi.bonacina@unige.ch (L. Bonacina).

1 These authors contributed equally to this work.

\section{Background}

Most of nanophotonics approaches (quantum dots, plasmonic nanoparticles (NP), up-conversion NP) for health applications present static optical properties (absorption bands, surface plasmon resonances) often in the UV-visible spectral region and do not fully allow for exploiting the tuning capabilities of new laser sources and their latest extensions in the infrared. To circumvent these limitations, a few research groups in the last years have introduced a new nanotechnology approach based on inorganic nanocrystals with non-centrosymmetric structures. Such nanomaterials present a very efficient nonlinear response, and can be easily imaged by their second harmonic generation 
(SHG) in multi-photon imaging platforms. ${ }^{1-6}$ Such harmonic NP (HNP) do not suffer from conventional optical limitations such as photobleaching and blinking allowing long-term monitoring of developing tissues. ${ }^{5,7}$ Several HNP have been recently synthesized and tested for biological applications. ${ }^{4-7}$ Particular care should be paid to assess the ability of these NP to reach intracellular targets without causing major interferences to the cell metabolism. In this context, this subcellular targeting becomes increasingly important as key parameters for the understating of complex events in living cells. ${ }^{8,9}$ In fact, the possibility to freely change detection wavelength can be exploited for subtle co-localization studies with organelle-specific dyes, as the signal from NP can be freely shifted to avoid overlap with fluorophores emission bands. However, one aspect that is not fully understood and ${ }^{10}$ remains uncertain is how nanomaterials interact with cellular interfaces such as cytoskeletal membranes since it is known that small alterations in their physicochemical properties can drastically influence the cells-NP interactions, especially the uptake mechanisms. ${ }^{9,11}$ Therefore, lead-NP-candidate identification process based on high throughput screening as decision-making process is a prerequisite for the validation of new SHG NP for bio-imaging applications. Here we present a study based on $\mathrm{BiFeO}_{3}$ (bismuth ferrite, abbreviated as BFO) NP (BFO-NP), which were recently successfully introduced as photodynamic tools and imaging probes. ${ }^{12}$ Nonetheless, such is the technological novelty of this new group of materials that there is still a knowledge gap that requires the scientific community attention towards the investigation of the interaction at the cellular and subcellular levels. The opportunity of closing this gap is presented by providing the first thorough investigation on the effects of BFO-NP in cellular metabolism and uptake mechanisms. Toxicity and biocompatibility were assessed by automated high content screening, recording cytotoxicity, lysosomal mass and cell permeability, in line with previously published works. ${ }^{13,14}$ Cellular uptake was investigated by co-localizing the NP with specific fluorophores for cell membranes and endosomes. Moreover, in this paper we present for the first time to our knowledge the most efficient protocol for the coating of these HNP with poly(ethylene glycol) (PEG) derivatives to promote colloidal stability and biocompatibility in biological media, and to allow post-functionalization with bioactive molecules. ${ }^{15,16}$ In this context, the biocompatibility, cellular uptake and intracellular localization of free and PEG coated BFO-NP were compared.

\section{Methods}

Preparation of a polydisperse suspension of $B F O$

The starting BFO suspension ( $2 \mathrm{~mL}, 62.5$ wt \% in $\mathrm{ZrO} 2$ balls), provided by the company FEE (Germany) under a collaboration agreement, was diluted in EtOH (2 L) and ultra-sonicated for $12 \mathrm{~h}$. After 10 days sedimentation, the upper portion of the polydisperse suspension $(50 \mathrm{~mL})$ was taken and mixed with oleic acid $(4 \mathrm{~mL})$. The volatiles were removed under vacuum and the residue was weighed and suspended in EtOH to obtain a stock solution at $3.6 \mathrm{mg} / \mathrm{mL}$.

\section{Coating of $\mathrm{BFO}-\mathrm{NP}$}

BFO-NP (suspended in EtOH, $3.6 \mathrm{mg} / \mathrm{mL}, 583 \mu \mathrm{L}$ ) were diluted in EtOH:toluene:25\% aqueous ammonia (0.50:0.50:0.32 mL) and ultra-sonicated for $30 \mathrm{~min}$. PEG oligomers $\mathbf{1}$ and $\mathbf{2}^{14}$ (1:1 ratio, $100 \mathrm{mg}$ ) were added and the suspension ultra-sonicated at $40{ }^{\circ} \mathrm{C}$ for $16 \mathrm{hr}$. The suspension was reduced to a small volume and distributed in plastic tubes for dilution with a mixture of dichloromethane (DCM):EtOH:water (1:1:1, $1 \mathrm{~mL})$. After centrifugation (10 min, $13000 \mathrm{rpm})$, the aqueous layer, containing the excess of unreacted polymers, was removed and a mixture of EtOH:water $(1: 1,0.5 \mathrm{~mL})$ was added to each plastic tube. The procedure was repeated 5 times to obtain a pure suspension of coated BFO-NP in DCM. After evaporation of DCM and addition of $\mathrm{EtOH}$, the BFO-NP concentration was calculated by measuring the turbidity of the solution by spectrometry at $600 \mathrm{~nm}$ (Synergy HT) and by comparing the values with a standard-curve prepared using the stock solution at $3.6 \mathrm{mg} / \mathrm{mL}$.

\section{Characterization of uncoated and coated BFO-NP}

Advanced physico-chemical characterization of uncoated and coated BFO-NP was recently performed and reported. In the present work, BFO-NP were characterized by dynamic light scattering (DLS) using a Zetasizer NanoZ (Malvern) for determination of mean hydrodynamic volume and zeta potential. Suspension of uncoated or coated BFO-NP $(20 \mu \mathrm{L})$ was diluted in $1 \mathrm{~mL}$ of distilled water. Acetic acid $(100 \mu \mathrm{L})$ was added and the resulting suspensions were ultra-sonicated for $30 \mathrm{~min}$ and analysed by DLS.

\section{Nanoparticles characterization in biological media}

The physico-chemical characterization of the NP was carried out by nanoparticle tracking analysis (NTA, Nanosight NS500). BFO-NP at $25 \mu \mathrm{g} / \mathrm{mL}$ were vortexed for $5 \mathrm{~s}$ to disperse the particles and then diluted at $1 \mu \mathrm{g} / \mathrm{mL}$ in different solutions $(0.22 \mu \mathrm{m}$ filtered): diethylpyrocarbonate (DEPC) water, Dulbecco's Modified Eagle Medium (DMEM), Roswell Park Memorial Institute (RPMI) and Ham's F-12 K (Kaighn's) Medium (F12K) culture media, and their supplemented form with $10 \%$ fetal bovine serum (FBS). Three culture media were chosen since commonly utilised for the in vitro culture cell models selected. The different dispersions were then analyzed via NTA for the physico-chemical characterization measurement of hydrodynamic radius and polydispersity index (PDI) at room temperature of BFO-NP. All measurements were carried out three times at physiologically relevant $\mathrm{pH}(\mathrm{pH}=7.4)$ and means and standard deviations (SD) were calculated. Quality assurance over the measurements carried out was guaranteed by the adoption of Quality Nano (QNano, FP7 project) standard operating procedures (SOPs), which have been developed as part of large inter laboratory comparative study focused on nanoparticle physico-chemical characterization. ${ }^{17}$

\section{Cell model and culturing conditions}

Human lung-derived A549 and NCI-H520 cancer cell lines and human monocytic THP-1 cell line are available from ATCC 
(American Tissue Culture Collection, Manassas, VA, USA). A549 were grown in DMEM medium containing $4.5 \mathrm{~g} / \mathrm{L}$ glucose, $10 \% \mathrm{FBS}$ and penicillin/streptomycin (PS) in a $37{ }^{\circ} \mathrm{C}$ incubator with $5 \% \mathrm{CO}_{2}$ at $95 \%$ humidity. NCI-H520 and THP-1 were grown in complete Roswell Park Memorial Institute (RPMI) 1640 medium supplemented with 10\% FCS and PS in a $37{ }^{\circ} \mathrm{C}$ incubator with $5 \% \mathrm{CO}_{2}$ at $95 \%$ humidity. For differentiation into macrophages, THP-1 cells were plated at a density of $20^{\prime} 000$ cells $/ \mathrm{cm}^{2}$ in RPMI 1640 supplemented with $10 \%$ FBS and $100 \mathrm{ng} / \mathrm{mL}$ phorbol 12-myristate 13-acetate (PMA, Sigma-Aldrich) for $72 \mathrm{~h}$. Differentiated THP-1 cells adhered to the bottom of the wells.

\section{Fluorescent staining for cellular imaging}

The cells (20'000 cells $/ \mathrm{cm}^{2}$ ) were grown for $48 \mathrm{~h}$ or $72 \mathrm{~h}$ for activated PMA THP-1 cells, in a 24 well plate containing one rod-shaped microscope slide (BD Falcon). After this time cells were exposed to BFO-NP at $25 \mu \mathrm{g} / \mathrm{mL}$ or to vehicle (ethanol) at indicated time. For endosomes imaging the cell layers were exposed for $2 \mathrm{~h}$ to $15 \mu \mathrm{g} / \mathrm{mL}$ of the fluorescent probe FM1-43FX (Invitrogen). After incubation, the cells were fixed in $4 \%$ formaldehyde in PBS for $30 \mathrm{~min}$, subsequently washed once in $0.1 \%$ Triton X-100 in PBS and twice in PBS, then maintained in formaldehyde. For membrane staining, cells were fixed and then exposed to $0.1 \mu \mathrm{g} / \mathrm{mL}$ Nile Red (Invitrogen) in PBS for $5 \mathrm{~min}$, then rinsed twice in PBS and maintained in formaldehyde. Cell nuclei were stained with 4',6'-diamidino-2phenylindole (DAPI).

\section{Multiphoton laser scanning microscopy}

Samples were observed using a Nikon multiphoton inverted microscope (A1R-MP) coupled with a Mai-Tai tunable Ti:sapphire oscillator from Spectra-Physics (100 fs, $80 \mathrm{MHz}$, 700-1000 nm). A Plan APO $40 \times$ WI N.A. 1.25 objective was used to focus the excitation laser and to epi-collect the SHG signal and dye markers fluorescence. Nanoparticles and fluorescent dyes (FM1-43FX and Nile Red) were excited at $790 \mathrm{~nm}$ and observed through tailored pairs of dichroic mirrors and interferometric filters (Semrock, FF01- 395/11-25 for SH, FF01-607/70-25 for fluorescence). Statistics were calculated by averaging measured values from samples between 40 and 170 cells per condition for cell labelling and between 8 and 100 cells per condition for co-localization measurements. Cell labelling was computed by dividing the number of cells labelled by at least one nanoparticle to the total number of cells in the microscope field of view and expressed as \% of total cells. Co-localization with endosomes was estimated by counting the number of nanoparticles co-localizing with the fluorescence signal from cell membrane dye FM1-43FX-and dividing this value by the total number of nanoparticles present inside each cell. The result of this procedure is expressed as \% of all particles internalized in each cell.

\section{In vitro dose and exposure endpoint determination}

Cytotoxicity on three-cell line models was investigated in vitro after $24 \mathrm{~h}$ and $72 \mathrm{~h}$ incubation with BFO-NP. Following standardization of the BFO preparation protocols, all were injected into 96 well plates to a final volume of $200 \mu \mathrm{L} /$ well.A549, NCI-H520 and activated PMA THP-1 cells were initially plated at a cell density of $10^{\prime} 000 \mathrm{cell} / \mathrm{cm}^{2}$ and incubated with $1.0,2.5,5.0$, 7.5 or $10 \mu \mathrm{g} / \mathrm{mL}$ of uncoated and coated BFO-NP for $24 \mathrm{~h}$ and $72 \mathrm{~h}$ in a $37{ }^{\circ} \mathrm{C}$ incubator with $5 \% \mathrm{CO}_{2}$ at $95 \%$ humidity. Experiments were repeated three times, using triplicate wells each time for each formulation tested. Positive and negative controls were also included into each experiment in order to quantify the extent of toxicity response induced by each particle. Three positive controls were Valinomycin (VAL, Fisher Scientific) (final concentration $120 \mu \mathrm{M}$ ) to measure changes in mitochondrial transmembrane potential, Tacrine (TAC, Sigma-Aldrich) (final concentration $100 \mu \mathrm{M}$ ) to measure changes in lysosomal mass $/ \mathrm{pH}$ and Quantum Dots (CdSe) (final concentration at $1 \mu \mathrm{M}$ ) to measure nanoparticle-induced uptake. ${ }^{14}$ After $24 \mathrm{~h}$ and $72 \mathrm{~h}$ incubation, cells were washed in phosphate-buffered saline solution (PBS) at pH 7.4 and fixed in 3\% paraformaldehyde (PFA). A multiparametric cytotoxicity assay was performed using the Cellomics ${ }^{\circledR}$ HCS reagent HitKitTM as per manufacturer's instructions (Thermo Fisher Scientific Inc.). For each experiment, each plate well was scanned and acquired in a stereology configuration of 6 randomly selected fields. In total, each endpoint data plot in the heatmaps represents the analysis of an average of 270,000 cells $=3$ runs $\times 3$ triplicates $\times 6$ fields $\times 5000$ cells (on average from $\mathrm{t}=0 \mathrm{~h}$ ). Images were acquired at $10 \mathrm{x}$ magnification using three detection channels with different excitation filters. These included a DAPI filter (channel 1), which detected blue fluorescence of the Cellomics ${ }^{\circledR}$ Hoechst 33342 probe indicating nuclear intensity at a wavelength of $461 \mathrm{~nm}$; FITC filter (channel 2 ), which detected green fluorescence of the Cellomics ${ }^{\circledR}$ cell permeability probe indicating cell permeability at a wavelength of $509 \mathrm{~nm}$ and a TRITC filter (channel 3), which detected the lysosomal mass and $\mathrm{pH}$ changes of the Cellomics ${ }^{\circledR}$ LysoTracker probe with red fluorescence at a wavelength of $599 \mathrm{~nm}$.

\section{Statistical analysis}

Response of each cell type to the coated and uncoated BFO-NP was analysed by 2-way ANOVA with Bonferroni post-test analysis. A $P$-value $<0.05$ was considered to be statistically significant. In this work we are comparing 4 cell parameters associated with the cytotoxicity response of 3 cell lines exposed to uncoated or coated BFO, at 5 doses, with 3 controls; therefore, the statistical value associated with our work carried out by High Content Screening and data mining is of significance. To visualize the data, KNIME (http:/KNIME.org, 2.0.3) data exploration platform and the screening module HiTS (http://code.google.com/p/hits, 0.3.0) were used. Knime is a modular open-source data manipulation and visualization programme, as previously reported. ${ }^{14,18-20}$ All measured parameters were normalized using the per cent of the positive controls. $\mathrm{Z}$ score was used for scoring the normalized values. These scores were summarized using the mean function as follows $\mathrm{Z}$ score (x-mean)/SD, as from previous work. ${ }^{14,18-20}$ Heatmaps graphical illustration in a colorimetric gradient table format was adopted as the most suitable schematic representation to report on any statistical significance and variation from normalized controls based on their $\mathrm{Z}$ score value. Heatmap tables illustrate the range 


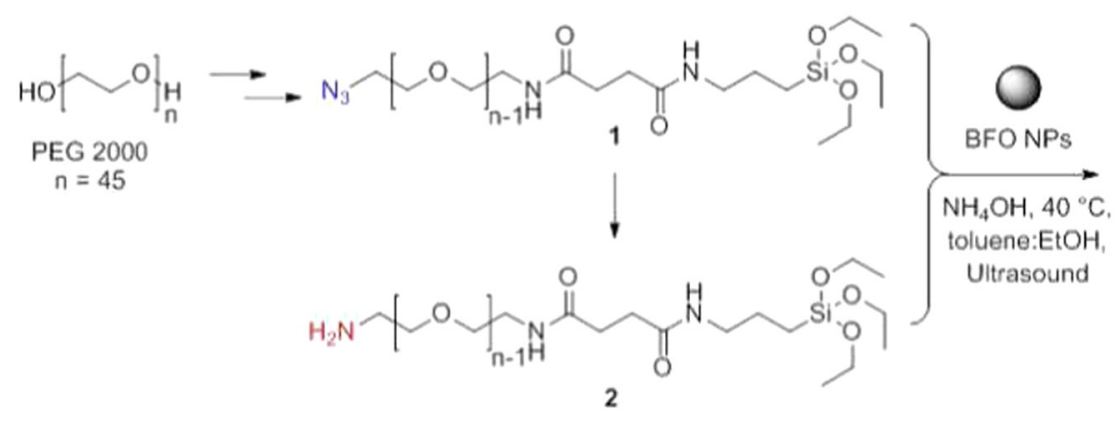

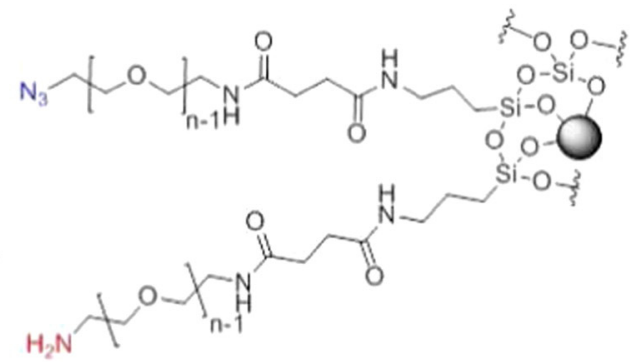

PEG-BFO-NP

Figure 1. Synthesis of coated BFO-NP. PEG oligomer (1) and PEG oligomer (2) were synthetized from PEG 2000 (MW 2000 Da) as published, ${ }^{15}$ then used for the coating of BFO-NP.

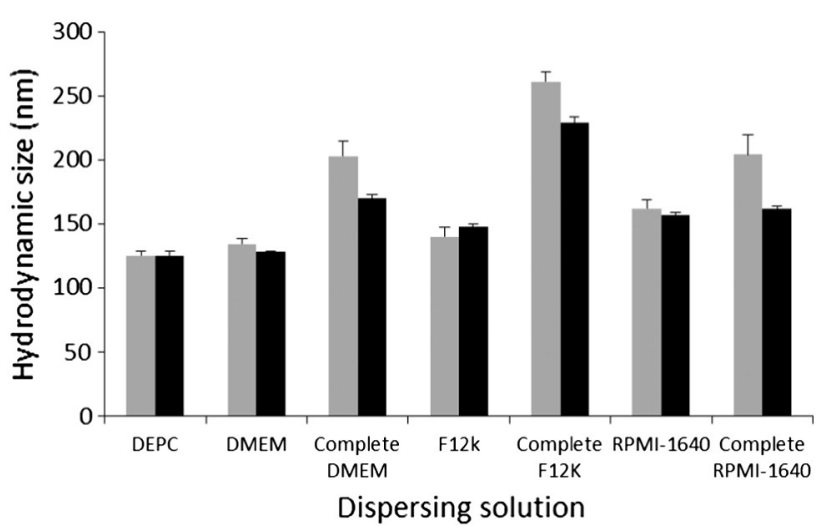

Figure 2. Nanoparticle tracking analysis of U-BFO-NP (light grey bars) and PEG-BFO-NP (dark grey bars) in biological media. Complete: media supplemented with $10 \%$ foetal bovine serum (FBS); DEPC: diethylpyrocarbonate; DMEM: Dulbecco's Modified Eagle Medium; F12k: Ham's F-12 K (Kaighn's) Medium; RPMI-1640: Roswell Park Memorial Institute 1640. Size distribution of BFO-NP after dispersion at $1 \mu \mathrm{g} / \mathrm{mL}$ in DEPC water solution (DEPC), DMEM medium (DMEM), DMEM medium supplemented with $10 \%$ FBS (complete DMEM), F12k medium (F12k), F12k medium supplemented with 10\% FBS (complete F12k), RPMI 1640 medium (RPMI-1640) and RPMI 1640 medium supplemented with $10 \%$ FBS (complete RPMI-1640). All measurements were carried out three times at $\mathrm{pH} 7.4$, then means and standard deviations (SD) were calculated.

of variation of each quantified parameter from the minimum (green) through the mean (yellow) to the maximum (red) according to the parameter under analysis.

\section{Haemolysis assay}

Fresh human blood in lithium heparin-containing tubes was obtained from leftovers of analytical blood with normal values. The plasma was removed by centrifugation for $10 \mathrm{~min}$ at $2500 \mathrm{rpm}$ and the blood cells were washed three times with sterile isotonic PBS solution, then diluted 1:10 in PBS. Cell suspensions $(300 \mu \mathrm{L})$ were added to $1200 \mu \mathrm{L}$ of each solution containing NP or chemicals in human plasma or PBS at indicated concentrations. Nanopure water $(1200 \mu \mathrm{L})$ was used as a positive control and human plasma or PBS $(1200 \mu \mathrm{L})$ were used as negative controls. The mixtures were gently mixed then kept for $2 \mathrm{~h}$ at RT, centrifuged for $2 \mathrm{~min}$ at $4000 \mathrm{rpm}$ and the absorbance of the upper layers was measured at $540 \mathrm{~nm}$ in an absorbance multi-well plate reader (Synergy HT). The percentage of haemolysis of the samples was calculated by dividing the difference in absorbance between the samples and the negative control by the difference in absorbance between the positive and negative controls. Experiments were conducted in triplicate wells and repeated twice. Means + SD were calculated.

\section{Results}

As previously stated, this work presents for the first time several important aspects relevant to the translation of BFP-NP into a novel biomedical imaging probe for advanced diagnostic screening.

\section{Coating and characterization of BFO-NP}

The presence of reactive hydroxyl groups at the surface of BFO-NP facilitates the surface coating chemistry. Modified poly(ethylene glycol) (PEG) 2000 (molecular weight of $2000 \mathrm{~g} /$ mol, 45 units) containing silane anchoring groups and reactive functionalities were synthetized as previously published ${ }^{15}$ and used to perform covalent coating via silane ( $\mathrm{Si}$ ) ligation (Figure 1).

A previously published study, by some of the authors, focused on the surface coating and post-functionalization of metal oxide NP such as iron oxide NP. ${ }^{15}$ In this work, BFO-NP were treated with an equimolar mixture of $\alpha$ - triethoxysilyl- $\omega$-azido and $\alpha$ - triethoxysilyl- $\omega$-amino PEG oligomers $\mathbf{1}$ and $\mathbf{2}$, prepared from linear PEG 2000, in the presence of aqueous ammonia. ${ }^{15}$ Ultra-sonication at $40{ }^{\circ} \mathrm{C}$ for 16 hours, followed by repetitive cycles of decantation/centrifugation into 1:1:1 DCM:EtOH:water resulted in coated BFO-NP (PEG-BFO-NP), which were suspended in EtOH for further characterization. Efficient coating was proved by FT-IR analysis (Supplementary Figure 1). Size and surface charge characteristics of uncoated BFO-NP (U-BFO-NP) and PEG-BFO-NP were measured using DLS and zeta potential techniques as previously described. ${ }^{15}$ Upon coating, the zeta potential value shifted from $-29.0 \pm$ $1.3 \mathrm{mV}$ to $-9.8 \pm 0.3 \mathrm{mV}$ and the mean hydrodynamic diameter decreased from $128.8 \pm 11.2 \mathrm{~nm}$ to $96.1 \pm 8.3 \mathrm{~nm}$. The decrease in the hydrodynamic diameter of coated BFO-NP can be attributed to a better dispersion of the NP in the solvent as a result of a possible colloidal stabilisation. ${ }^{21}$ 

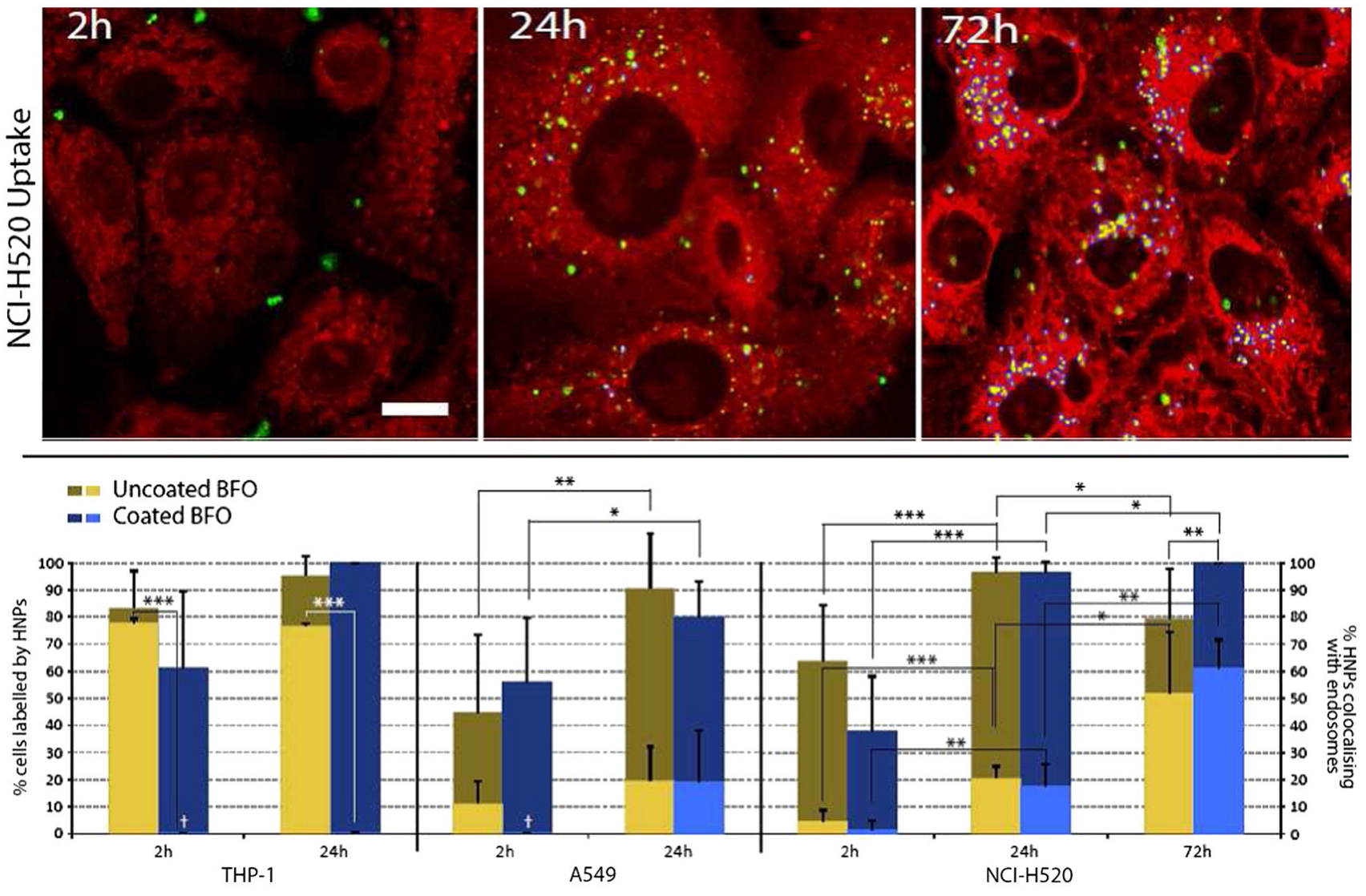

Figure 3. BFO-NP uptake in human-derived cell lines.First row: NCI-H520 cancer cells exposed to PEG-BFO-NP at $25 \mu \mathrm{g} / \mathrm{mL}$ for $2 \mathrm{~h}, 24 \mathrm{~h}$ and $72 \mathrm{~h}$. Green: SHG signal of BFO-NP; yellow: co-localization between the probe for lipid membranes and the particles; blue circles: highlight of HNP co-localizing with FM1-43FX. Scale bar: $10 \mu \mathrm{m}$.Second row: \% of labelled A549, NCI-H520 and THP-1 cells exposed to U-BFO-NP (brown bars) or PEG-BFO-NP (blue bars) at $25 \mu \mathrm{g} / \mathrm{mL}$ for $2 \mathrm{~h}, 24 \mathrm{~h}$ or $72 \mathrm{~h}$ (only NCI-H520). Statistical comparisons were done using a Student's $t$ test: ${ }^{*} P<0.05,{ }^{* *} P<0.01, * * * P<0.001$. $\dagger:$ no HNP co-localizing with endosomes were observed.

\section{BFO-NP stability and characterization in biological media}

For both uncoated and coated BFO-NP, extended physicochemical characterization was carried out after ultracentrifugation and re-dispersion in ultrapure deionized water prior to incubation into relevant biological dispersing media. The two devised BFO-NP potential probes were also characterized by NTA, aiming at the identification of hydrodynamic radii, the colloidal and the aggregation stabilities at physiologically relevant conditions (Figure 2). Interestingly, U-BFO-NP formed aggregates in biological relevant media, particularly when the media were supplemented with serum. PEG-BFO-NP resulted in a decrease of aggregate formation, and thus a better stability of the suspension. This finding was further confirmed after $24 \mathrm{~h}$ incubation (Supplementary Figure 2). Through this process it was also possible to select the culture media to utilise for dispersion during the biological studies undertaken.

\section{Interaction between BFO-NP and human derived cells}

The interactions between U-BFO-NP and PEG-BFO-NP were then studied in three human-derived cell lines, one human adenocarcinoma cell line (A549) derived from alveolar epithelial type II cells, one lung squamous carcinoma cell line
$(\mathrm{NCI}-\mathrm{H} 520)^{6}$ and one human acute monocytic leukemia cell line (THP-1) as a model for macrophages. ${ }^{22}$ These experiments were aimed at observing the interaction between particles and cell membranes in vitro and to explore the uptake of particles in cells, particularly the endocytic pathways. Indeed, endocytosis represents one of the main internalization mechanisms of NP in cells, ${ }^{11,15,23}$ particularly in macrophages. ${ }^{23,24}$ For the quantification of particles associated with the plasma membranes and internalized into the cytoplasm, cells were exposed to BFO-NP, fixed and labelled with a fluorescent probe used to stain membranes and lipids. ${ }^{25}$ Exposure time of $2 \mathrm{~h}$ and $24 \mathrm{~h}$ were chosen since considered representative minimum and maximum exposure time for diagnostic applications; whereas $72 \mathrm{~h}$ was identified as a critical time point for cytotoxicity response. HNP uptake by endocytosis was observed by co-localizing the SHG signal with a molecular probe specifically internalized in the endosomes. ${ }^{26}$ It is worth pointing out that for the following uptake quantification we purposely adopted an extremely strong criterion (a cell is labelled if at least one particle is in contact with it). Even with this strict definition, we observed significant cell-dependent responses in the uptake between $2 \mathrm{~h}$ and $24 \mathrm{~h}$, especially for macrophages. In fact, a consistent uptake by these latter cells was observed for the two diagnostically relevant 


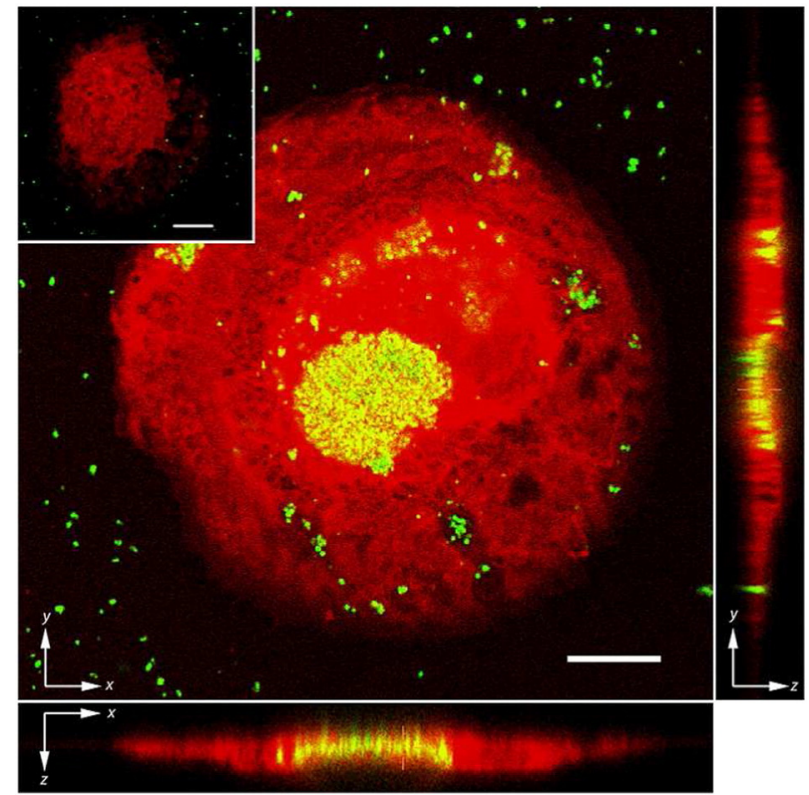

Figure 4. Uptake of uncoated BFO-NP by activated THP-1 cells. Two-photon microscopy image of an individual THP-1 cell exposed for $24 \mathrm{~h}$ at $25 \mu \mathrm{g} / \mathrm{mL}$ U-BFO-NPs. Red: FM1-43FX fluorescence. Green: SH emission by NPs. Three dimensional information are obtained by z-stacking ov several images, the yz (right) and xz slice (bottom) views corresponds to cuts along the directions indicated by the yellow cross. Inset: Image of a THP-1 cell after $24 \mathrm{~h}$ exposure to $25 \mu \mathrm{g} / \mathrm{mL}$ of PEG-BFO-NP. Scale bars: $10 \mu \mathrm{m}$. One can see how the presence of PEG surface coating on NPs prevents NPs internalization by THP-1 cells.

exposure times ( $2 \mathrm{~h}$ and $24 \mathrm{~h}$ ), whereas in human lung-derived NCI-H520 cancer cells the uptake of the HNP was still observable after $72 \mathrm{~h}$ incubation (as shown in Figure 3 low panel). Thus, from the results presented cell-dependent and time-dependent internalisation of the HNP into the three cell models adopted could be shown, as comprehensively presented between Figure 3 and the multiparametric heatmaps Figure 5. And more, it also emerges that consistency in the HNP internalization in human lung-derived cancer cells resulted to be time dependent (Figure 3). Indeed, HNP generally adhere to the cell membrane after $2 \mathrm{~h}$ and are then internalized when increasing exposure time. After $72 \mathrm{~h}$ exposure, BFO-NP formed aggregates in intracellular organelles, such as lysosome or endosomes. We did not observe a statistically significant difference in labelling between PEG-BFO-NP and U-BFO-NP after $2 \mathrm{~h}$ or $24 \mathrm{~h}$ exposure (Figure 3). Interestingly the interaction HNP with immune THP-1 cells was fairly rapid, as more than $80 \%$ of cells were found to have internalized at least one particle already after $2 \mathrm{~h}$ exposure. Such finding is in line with previous observations, since it is known that THP-1 phagocytic cells rapidly envelop and digest extraneous objects as initial response to infection or foreign body invasion. ${ }^{10,22,27}$ Conversely, the labelling of A549 and NCI-H520 cells showed a clear exposure dependent behaviour. After $72 \mathrm{~h}$, the number of labelled cells was significantly higher in the presence of PEG-BFO-NP than with U-BFO-NP (Figure 3), suggesting the existence of an exocytosis mechanism for the uncoated BFO-NP as already observed and reported for others metallic NP. ${ }^{28,29}$ In macrophages, the uptake of uncoated particles is mainly endocytic-dependent, whereas the PEGylation of BFO-NP completely reduced the endocytic-mediated uptake (Figure 3). This mechanism of particle internalization was confirmed by multiphoton laser scanning microscopy z-stack analysis of differentiated THP-1, which showed clusters of BFO-NP into the cytoplasm of cells having internalized uncoated BFO-NP after $24 \mathrm{~h}$ exposure (Figure 4) as opposed to the behaviour observed with coated BFO-NP, which showed a reduced uptake. In the lung-derived cancer cell lines only a weak fraction of HNP co-localized with the endosomes after $2 \mathrm{~h}$ and $24 \mathrm{~h}$ exposure, nonetheless this portion increased for the NCI-H520 cells after $72 \mathrm{~h}$ exposure to the particles.

\section{Multiparametric cytotoxicity evaluation of BFO-NP}

The cytotoxicity of uncoated and coated BFO-NP was investigated in vitro in order to address any cell-dependent and time-dependent toxicity aspects associated to the two human lung-derived NCI-H520 and A549 cancer cell lines and the macrophage-derived THP-1 cells. Multiparametric cytotoxicity was qualitatively and quantitatively measured after $24 \mathrm{~h}$ and $72 \mathrm{~h}$ exposure to increasing concentrations $(1,2.5,5,7.5$ and $10 \mu \mathrm{g} / \mathrm{mL}$ ) of HNP particles. Experiments were repeated three times, using triplicate wells each time for each formulation tested, as shown in Supplementary Table 1. Three key parameters of toxicity-attributed phenomena were analysed during this assay: cell number, lysosomal mass and $\mathrm{pH}$, and cell membrane permeability, as previously reported. ${ }^{14}$ Indeed, it is known that some toxins can interfere with the cell's functionality by affecting the $\mathrm{pH}$ of organelles such as lysosomes and endosomes, or by causing an increase in the number of lysosomes present. ${ }^{14,30}$ Cell membrane permeability changes can be measured as enhancement of cell membrane damage and decreased cell viability as result of cell nuclear-staining counting reduction. ${ }^{14,31}$ Moreover, three positive controls were introduced in this analysis: CdSe quantum dots (QD), which are established toxic NP, ${ }^{14}$ Valinomycin (Vac), an inducer of energy-dependent mitochondrial swelling causing cell membrane permeability ${ }^{32}$ and Tacrine (Tac), a reversible cholinesterase inhibitor which causes increases in cellular lysosome content and lysosomal mass. ${ }^{33}$ The rate of cell viability and proliferation was assessed by automated quantitative analysis of the nuclear count and cellular morphology; in parallel to that the fluorescent staining intensities reflecting cell permeability and lysosomal mass $/ \mathrm{pH}$ changes were also quantified for each individual cell measured within each imaged field and then colorimetrically normalised against the respective controls (Figure 5). A multiparametric analysis of the cellular responses allowed the identification of the different toxicity levels between the two BFO-NP. In general, BFO-NP in concentration range of $1-10 \mu \mathrm{g} / \mathrm{mL}$ showed cell-dependent cytotoxicity on the cell models adopted. In agreement with previous results, THP-1 cells are the most affected cells between the three cell lines since these are the first line immune response to any foreign objects such as nanoparticles. For both PEG-BFO-NP and U-BFO-NP a timedependent and dose-dependent toxicity was measured. However, 


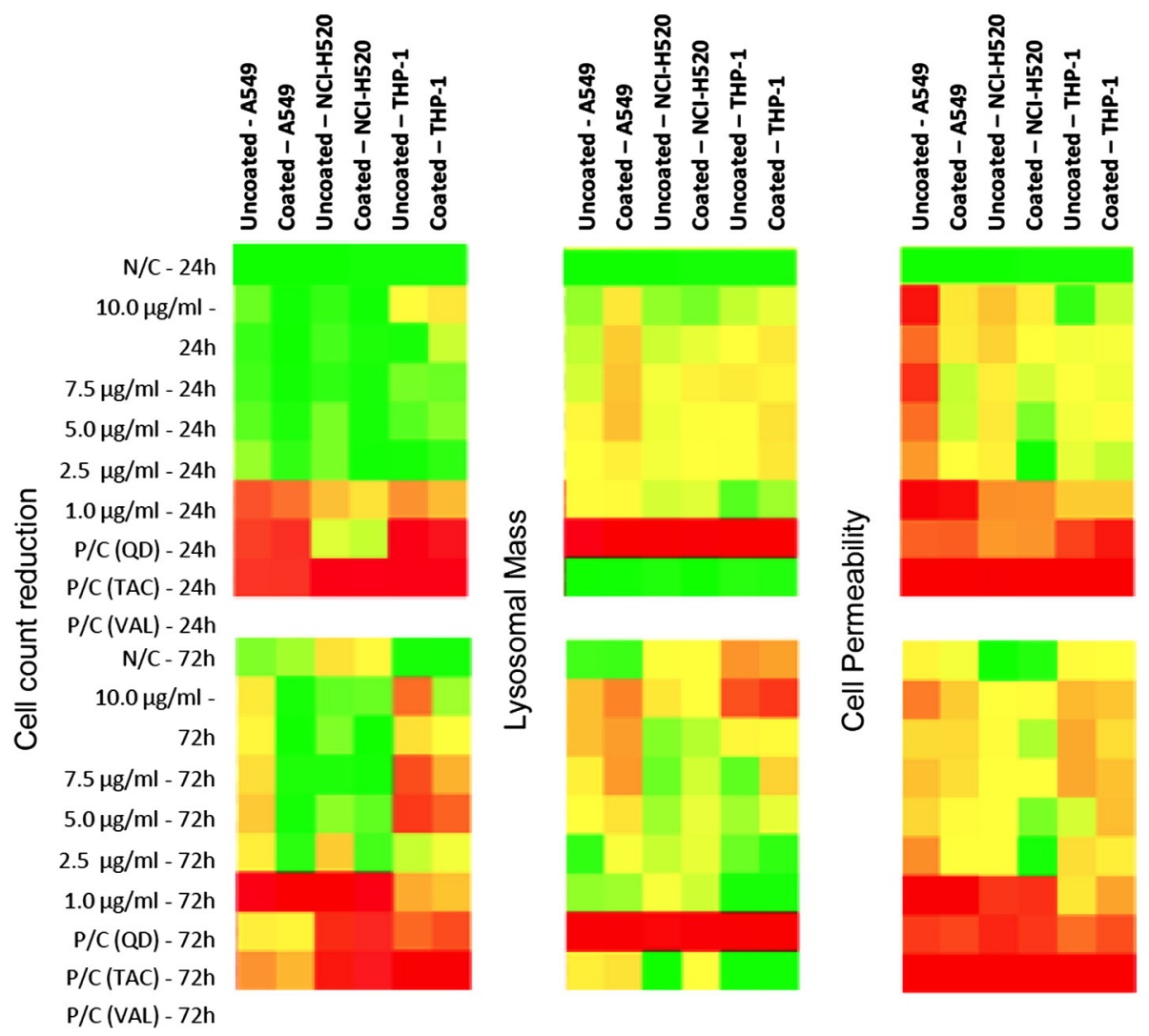

Figure 5. Heatmaps tables illustrating toxicity indicated parameters of BFO-NP in human-derived cell lines. Heatmaps were generated from the analysis of $\mathrm{n}=3$ experiments, each with triplicate wells for each of the parameters under investigation: cell count, lysosomal mass, cell permeability and nuclear area. Colorimetric gradient table spans from: Dark green: lower than $15 \%$ of maximum value measured; Bright green $=30 \%$; Yellow $=50 \%$; Bright orange: $60 \%$; Dark orange $=75 \%$; Red higher than $75 \%$ of maximum value. N/C untreated controls (negative) and P/C as positive controls such as QD: quantum dots, TAC: Tacrine, VAL: Valinomycin). Heatmap values are normalised using the per cent of the positive controls and, Z score was calculated as described in the statistical analysis section.

these effects were more pronounced when cells were exposed to uncoated particles. Among the parameters measured during the NP comparison, the cell permeability parameter showed the most pronounced difference, suggesting that BFO-NP, particularly uncoated particles, interfered mainly with the physiology of the plasma membrane, as also shown by the Vac response as positive control for cellular permeability. PEG-BFO-NP got stored in lysosomal compartments as from lysosomal concentration response, which was also comparable to the response to Tac used positive control for lysosomal response.

\section{Haemolysis assay}

After having assessed the colloidal stability of PEGcoated BFO-NP in biological media, the interactions with cell membranes were tested by assessing their haemolytic effect on human red blood cells (HRBc) according to established protocols. ${ }^{6,34}$ Haemolysis is defined as the destruction of red blood cells and it is regarded as a key parameter for the evaluation of NP biocompatibility. ${ }^{6,34,35}$ NP can exert haemolytic effect by electrostatic interactions with membrane proteins or by other NP-specific mechanisms such as the generation of reactive oxygen species (ROS), causing irreversible damage to cells. The assessment of the haemolysis of uncoated BFO-NP showed a weak haemolytic potential (Figure 6), comparable to that of metallic NP observed in other bio-assays. ${ }^{35,36}$ Upon PEG coating, this potential was significantly reduced $(P<0.001$, Figure 6$)$. These statistically significant results proved that PEGylation of the metal oxide core contributed to the reduction of the interaction between cell membranes and particles surface.

\section{Discussion}

BFO-NP are a class of nanomaterials with unique optical and physical properties attracting the interest of researchers for several technological applications, in particular related to their multiferroics nature. We have shown recently their potential in the context of advanced non-linear optical imaging. ${ }^{37}$ Recently BFO-NP were employed in vitro in a pilot study where they proved to be able to locally induce DNA damages by deep UV generation. ${ }^{12}$ The cytotoxicity, haemolytic response and internalization mechanisms evidence here reported for coated BFO-NP suggested good biocompatibility and a great potential 


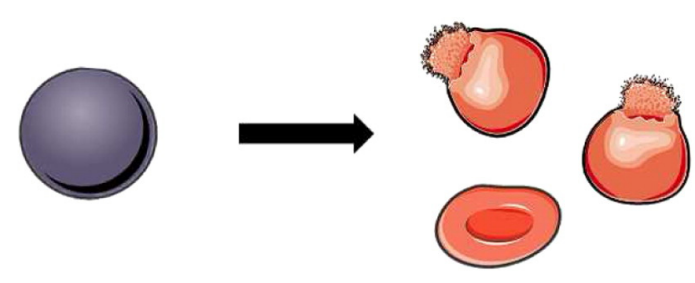

U-BFO-NP

$$
7.1 \%+0.4
$$
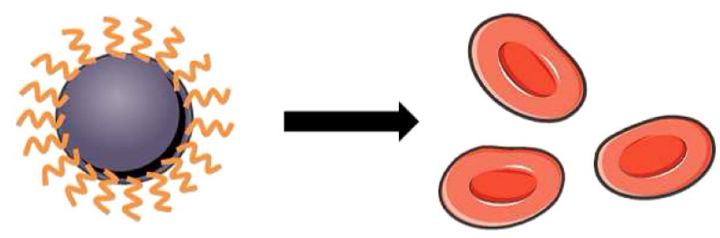

PEG-BFO-NP

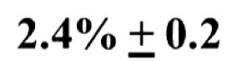

Figure 6. Haemolytic effect of U-BFO-NP and PEG-BFO-NP.HBRc were exposed to NP $(25 \mu \mathrm{g} / \mathrm{mL})$ for $2 \mathrm{~h}$. Results were expressed as \% of haemolysis of NP-exposed HRBc compared to unexposed cells. Results are the means $+\mathrm{SD}$ of triplicates of two independent experiments. Values of U-BFO-NP were compared to those of PEG-BFO-NP by a Student's $t$ test $(P<0.001)$. This picture was done using Servier Medical Art images.

for biomedical imaging in diagnostic applications, e.g. flow cytometry. ${ }^{38}$ Therefore, the aim of this work was, for the first time, to present the behaviour of these particles in their uncoated or PEGylated form, focusing on relevant exposure time point relevant to diagnostic applications. Such assessment comes timely after the first demonstrations of their interest for bio-imaging and selective photointeraction, as, due to their novelty, there is still a knowledge gap that requires the scientific community attention towards the investigation of their biological effect. NP must be coated with biocompatible polymers in order to stabilize the NP suspensions in biological media and to increase their biocompatibility. ${ }^{39,40}$ Coating with organic polymers also allows particles conjugation and functionalization with biologically-active ligands, such as targeting-specific ligands, therapeutic agents, peptides or antibodies. ${ }^{16,41}$ Here we present a method for the successful PEGylation of BFO-NP based on heterobifunctional PEG oligomers, for which a fast and convenient large scale synthesis protocol was recently published. ${ }^{16}$ PEG was selected as coating polymer due to its interesting properties for biomedical applications. Indeed, when compared to any other known polymer, PEG exhibits high hydrophilicity, low protein adsorption, low uptake by immune cells, and no toxicity properties. ${ }^{15,42,43}$ Our findings confirmed the important role of this kind of coating in terms of biocompatibility. Interestingly as presented here, PEG-BFO-NP are less toxic and their uptake in immune-responsive THP-1 cells is reduced. As a result of the multiparametric cytotoxicity evaluation, we hypothesize that BFO-NP toxicity is mainly mediated by electrostatic interactions with the surface of the particles and the cell membranes. The reduction of cytotoxicity observed upon coating is probably due to a steric barrier between the surface of the coated particles and the lipid membrane of cells. ${ }^{15,16,42}$ PEG-BFO-NP were internalized in intracellular organelles and they remained located into the cells sensibly longer than U-BFO-NP. This opens interesting ways for biomedical applications, which require stable incorporation of HNP in cells. In the future, more detailed studies about BFO-NP co-localization in cells are needed in order to better understand the pathways involved in the internalization of these particles. Moreover, more detailed assessment of the immune response associated with these particles, such as a cytokine profile after exposure to the particles, could be interesting for the elucidation of their biological effects since they are immune-responding to foreign body. ${ }^{44-47}$ However, this work confirmed the biocompatibility of BFO-NP and their utility as nanoprobes for biological - in particular diagnostics - applications.

\section{Appendix A. Supplementary data}

Supplementary data to this article can be found online at http://dx.doi.org/10.1016/j.nano.2014.12.018.

\section{References}

1. Bonacina L, Mugnier Y, Courvoisier F, Le Dantec R, Extermann J, Lambert $\mathrm{Y}$, et al. Polar Fe(IO3)(3) nanocrystals as local probes for nonlinear microscopy. Appl Phys B Lasers Opt 2007;87:399-403.

2. Bonacina L. Nonlinear nanomedecine: harmonic nanoparticles toward targeted diagnosis and therapy. Mol Pharm 2013;10:783-92.

3. Extermann J, Bonacina L, Cuna E, Kasparian C, Mugnier Y, Feurer T, et al. Nanodoublers as deep imaging markers for multi-photon microscopy. Opt Express 2009;17:15342-9.

4. Magouroux T, Extermann J, Hoffmann P, Mugnier Y, Le Dantec R, Jaconi ME, et al. High-speed tracking of murine cardiac stem cells by harmonic nanodoublers. Small 2012;8:2752-6.

5. Pantazis P, Maloney J, Wu D, Fraser SE. Second harmonic generating (SHG) nanoprobes for in vivo imaging. Proc Natl Acad Sci US A 2010;107:14535-40.

6. Staedler D, Magouroux T, Hadji R, Joulaud C, Extermann J, Schwung S, et al. Harmonic nanocrystals for biolabeling: a survey of optical properties and biocompatibility. ACS Nano 2012;6:2542-9.

7. Zielinski M, Oron D, Chauvat D, Zyss J. Second-harmonic generation from a single core/shell quantum dot. Small 2009;5:2835-40.

8. Chou LY, Ming K, Chan WC. Strategies for the intracellular delivery of nanoparticles. Chem Soc Rev 2011;40:233-45.

9. Kim TH, El-Said WA, An JH, Choi JW. ITO/gold nanoparticle/RGD peptide composites to enhance electrochemical signals and proliferation of human neural stem cells. Nanomedicine 2013;9:336-44.

10. Auwerx J. The human leukemia-cell line, Thp-1 - a multifaceted model for the study of monocyte-macrophage differentiation. Experientia 1991; 47:22-31.

11. Herd H, Daum N, Jones AT, Huwer H, Ghandehari H, Lehr CM. Nanoparticle geometry and surface orientation influence mode of cellular uptake. ACS Nano 2013;7:1961-73.

12. Staedler D, Magouroux T, Passemard S, Schwung S, Dubled M, Schneiter GS, et al. Deep UV generation and direct DNA photointeraction by harmonic nanoparticles in labelled samples. Nanoscale 2014;6:2929-36.

13. Mohamed BM, Verma NK, Prina-Mello A, Williams Y, Davies AM, Bakos G, et al. Activation of stress-related signalling pathway in human cells upon $\mathrm{SiO} 2$ nanoparticles exposure as an early indicator of cytotoxicity. J Nanobiotechnol 2011;9(29):1-14. 
14. Prina-Mello A, Crosbie-Staunton K, Salas G, Morales MD, Volkov Y. Multiparametric toxicity evaluation of SPIONs by high content screening technique: identification of biocompatible multifunctional nanoparticles for nanomedicine. IEEE Trans Magn 2013;49:377-82.

15. Hak S, Helgesen E, Hektoen HH, Huuse EM, Jarzyna PA, Mulder WJ, et al. The effect of nanoparticle polyethylene glycol surface density on liganddirected tumor targeting studied in vivo by dual modality imaging. ACS Nano 2012;6:5648-58.

16. Passemard S, Staedler D, Ucnova L, Schneiter GS, Kong P, Bonacina L, et al. Convenient synthesis of heterobifunctional poly(ethylene glycol) suitable for the functionalization of iron oxide nanoparticles for biomedical applications. Bioorg Med Chem Lett 2013;23:5006-10.

17. Hole P, Sillence K, Hannell C, Maguire CM, Roesslein M, Suarez G, et al. Interlaboratory comparison of size measurements on nanoparticles using nanoparticle tracking analysis (NTA). J Nanopart Res 2013;15:2101.

18. Mohamed BM, Verma NK, Davies AM, McGowan A, Crosbie-Staunton K, Prina-Mello A, et al. Citrullination of proteins: a common posttranslational modification pathway induced by different nanoparticles in vitro and in vivo. Nanomedicine (Lond) 2012;7:1181-95.

19. Movia D, Prina-Mello A, Volkov Y, Giordani S. Determination of spiropyran cytotoxicity by high content screening and analysis for safe application in bionanosensing. Chem Res Toxicol 2010;23:1459-66.

20. Movia D, Prina-Mello A, Bazou D, Volkov Y, Giordani S. Screening the cytotoxicity of single-walled carbon nanotubes using novel 3D tissuemimetic models. ACS Nano 2011;5:9278-90.

21. Gupta AK, Wells S. Surface-modified superparamagnetic nanoparticles for drug delivery: preparation, characterization, and cytotoxicity studies. IEEE Trans Nanobioscience 2004;3:66-73.

22. Kohro T, Tanaka T, Murakami T, Wada Y, Aburatani H, Hamakubo T, et al. A comparison of differences in the gene expression profiles of phorbol 12-myristate 13-acetate differentiated THP-1 cells and human monocyte-derived macrophage. J Atheroscler Thromb 2004;11:88-97.

23. Shukla R, Bansal V, Chaudhary M, Basu A, Bhonde RR, Sastry M. Biocompatibility of gold nanoparticles and their endocytotic fate inside the cellular compartment: a microscopic overview. Langmuir 2005;21:10644-54.

24. Lankoff A, Sandberg WJ, Wegierek-Ciuk A, Lisowska H, Refsnes M, Sartowska B, et al. The effect of agglomeration state of silver and titanium dioxide nanoparticles on cellular response of HepG2, A549 and THP-1 cells. Toxicol Lett 2012;208:197-213.

25. Lee Y, Yang I, Lee JE, Hwang S, Lee JW, Um SS, et al. Enhanced photocurrent generation by Forster resonance energy transfer between phospholipid-assembled conjugated oligoelectrolytes and Nile red. $J$ Phys Chem C 2013;117:3298-307.

26. Hansen GH, Rasmussen K, Niels-Christiansen LL, Danielsen EM. Endocytic trafficking from the small intestinal brush border probed with FM dye. Am J Physiol Gastrointest Liver Physiol 2009;297:G708-15.

27. Tsuchiya S, Yamabe M, Yamaguchi Y, Kobayashi Y, Konno T, Tada K. Establishment and characterization of a human acute monocytic leukemia cell line (THP-1). Int J Cancer 1980;26:171-6.

28. Chithrani BD, Chan WC. Elucidating the mechanism of cellular uptake and removal of protein-coated gold nanoparticles of different sizes and shapes. Nano Lett 2007;7:1542-50.

29. Jin H, Heller DA, Sharma R, Strano MS. Size-dependent cellular uptake and expulsion of single-walled carbon nanotubes: single particle tracking and a generic uptake model for nanoparticles. ACS Nano 2009;3:149-58.
30. Marquis BJ, Love SA, Braun KL, Haynes CL. Analytical methods to assess nanoparticle toxicity. Analyst 2009;134:425-39.

31. Jeng HA, Swanson J. Toxicity of metal oxide nanoparticles in mammalian cells. J Environ Sci Health A Tox Hazard Subst Environ Eng 2006;41:2699-711.

32. Lofrumento DD, La Piana G, Abbrescia DI, Palmitessa V, La Pesa V, Marzulli D, et al. Valinomycin induced energy-dependent mitochondrial swelling, cytochrome c release, cytosolic NADH/cytochrome c oxidation and apoptosis. Apoptosis 2011;16:1004-13.

33. Monteith DK, Theiss JC, Haskins JR, de la Iglesia FA. Functional and subcellular organelle changes in isolated rat and human hepatocytes induced by tetrahydroaminoacridine. Arch Toxicol 1998;72:147-56.

34. He Q, Zhang J, Shi J, Zhu Z, Zhang L, Bu W, et al. The effect of PEGylation of mesoporous silica nanoparticles on nonspecific binding of serum proteins and cellular responses. Biomaterials 2010;31:1085-92.

35. Choi J, Reipa V, Hitchins VM, Goering PL, Malinauskas RA. Physicochemical characterization and in vitro hemolysis evaluation of silver nanoparticles. Toxicol Sci 2011;123:133-43.

36. Zhao YN, Sun XX, Zhang GN, Trewyn BG, Slowing II, Lin VSY. Interaction of mesoporous silica nanoparticles with human red blood cell membranes: size and surface effects. ACS Nano 2011;5:1366-75.

37. Schwung S, Rogov A, Clarke G, Joulaud C, Magouroux T, Staedler D, et al. Nonlinear optical and magnetic properties of $\mathrm{BiFeO} 3$ harmonic nanoparticles. J Appl Phys 2014;116:114306, http://dx.doi.org/10.1063/1.4895836.

38. Geissbuehler M, Bonacina L, Shcheslavskiy V, Bocchio NL, Geissbuehler S, Leutenegger M, et al. Nonlinear correlation spectroscopy (NLCS). Nano Lett 2012;12:1668-72.

39. Lin MM, Kim HH, Kim H, Dobson J, Kim do K. Surface activation and targeting strategies of superparamagnetic iron oxide nanoparticles in cancer-oriented diagnosis and therapy. Nanomedicine (Lond) 2010;5:109-33.

40. Shah NB, Vercellotti GM, White JG, Fegan A, Wagner CR, Bischof JC. Blood-nanoparticle interactions and in vivo biodistribution: impact of surface PEG and ligand properties. Mol Pharm 2012;9:2146-55.

41. Gil PR, Parak WJ. Composite nanoparticles take aim at cancer. ACS Nano 2008;2:2200-5.

42. Perry JL, Reuter KG, Kai MP, Herlihy KP, Jones SW, Luft JC, et al. PEGylated PRINT nanoparticles: the impact of PEG density on protein binding, macrophage association, biodistribution, and pharmacokinetics. Nano Lett 2012;12:5304-10.

43. Verma A, Stellacci F. Effect of surface properties on nanoparticle-cell interactions. Small 2010;6:12-21.

44. Hanley C, Thurber A, Hanna C, Punnoose A, Zhang J, Wingett DG. The influences of cell type and $\mathrm{ZnO}$ nanoparticle size on immune cell cytotoxicity and cytokine induction. Nanoscale Res Lett 2009; 4:1409-20.

45. Lesniak A, Fenaroli F, Monopoli MR, Aberg C, Dawson KA, Salvati A. Effects of the presence or absence of a protein corona on silica nanoparticle uptake and impact on cells. ACS Nano 2012;6:5845-57.

46. Sayes CM, Reed KL, Warheit DB. Assessing toxicity of fine and nanoparticles: comparing in vitro measurements to in vivo pulmonary toxicity profiles. Toxicol Sci 2007;97:163-80.

47. Siglienti I, Bendszus M, Kleinschnitz C, Stoll G. Cytokine profile of iron-laden macrophages: implications for cellular magnetic resonance imaging. J Neuroimmunol 2006;173:166-73. 